Research note

\title{
The potential of SARS-CoV-2 antigen-detection tests in the screening of asymptomatic persons
}

\author{
Jonas Wachinger ${ }^{1, \dagger}$, Ioana Diana Olaru ${ }^{1,2, \dagger}{ }^{\dagger}$, Susanne Horner ${ }^{1}$, Paul Schnitzler ${ }^{1}$, \\ Klaus Heeg ${ }^{1}$, Claudia M. Denkinger ${ }^{1,3}$,* \\ 1) Center of Infectious Diseases, Heidelberg University Hospital, Heidelberg, Germany \\ 2) Clinical Research Department, Faculty of Infectious and Tropical Diseases, London School of Hygiene and Tropical Medicine, London, UK \\ 3) German Center of Infection Research, Site Heidelberg, Heidelberg, Germany
}

\section{A R T I C L E I N F O}

\section{Article history:}

Received 22 May 2021

Received in revised form

16 July 2021

Accepted 18 July 2021

Available online xxx

Editor: F. Allerberger

\section{Keywords:}

Ag-RDTs

Diagnostic yield

Positive predictive value

SARS-CoV-2

Screening

\begin{abstract}
A B S T R A C T
Objectives: The aim was to assess the performance of antigen-based rapid diagnostic tests (Ag-RDTs) for SARS CoV-2 when implemented for large-scale universal screening of asymptomatic individuals.

Methods: This study was a pragmatic implementation study for universal Ag-RDT-based screening at a tertiary care hospital in Germany where patients presenting for elective procedures and selected personnel without symptoms suggestive of SARS-CoV-2 were screened with an Ag-RDT since October 2020. Test performance was calculated on an individual patient level.

Results: In total, 49542 RDTs were performed in 27421 asymptomatic individuals over a duration of 5 and a half months. Out of 222 positive results, 196 underwent in-house confirmatory testing with PCR, out of which 170 were confirmed positive, indicating a positive predictive value of $86.7 \%$ (95\% CI 81.2 -91.1\%). Negative Ag-RDTs were not routinely tested with PCR, but a total of 94 cases of false negative Ag-RDTs were detected due to PCR tests being performed within the following 5 days with a median cycle threshold value of 33 (IQR 29-35).

Discussion: This study provides evidence that Ag-RDTs can have a high diagnostic yield for transmission relevant infections with limited false positives when utilized at the point of care on asymptomatic patients and thus can be a suitable public health test for universal screening. Jonas Wachinger, Clin Microbiol Infect 2021; :-1

(C) 2021 The Author(s). Published by Elsevier Ltd on behalf of European Society of Clinical Microbiology and Infectious Diseases. This is an open access article under the CC BY-NC-ND license (http:// creativecommons.org/licenses/by-nc-nd/4.0/).
\end{abstract}

\section{Introduction}

Several researchers and policy makers, supported by evidence from modelling studies, have recently argued to increase largescale screening for SARS-CoV-2 to curb transmission from patients with minimal or no symptoms [1-5]. Antigen detection point-of-care rapid diagnostic tests (Ag-RDTs) have shown very good sensitivity (88\%) in persons with high viral load (cycle threshold $(\mathrm{CT})<30$ ) along with high specificity (>99\%) [6,7]. With their favourable ease of use, rapid turnaround and good (although suboptimal) performance, Ag-RDTs meet the characteristics for a test for public health use and could allow for better control of transmission if implemented in well-designed universal screening strategies $[8,9]$. However, one frequently raised concern has been the potentially imperfect specificity, leading to large numbers of false positives when using Ag-RDTs in largescale screening strategies with low prevalence [10], which could conceivably disrupt workflows and undermine trust in the test. Furthermore, in a setting where high-risk persons are present (e.g., hospital), concerns exist regarding imperfect sensitivity leading to secondary cases and substantial morbidity and mortality. Data from large-scale screening implementation efforts that would allow to gauge diagnostic yield and issues with false positives are limited.

\footnotetext{
* Corresponding author. Claudia M. Denkinger, Center of Infectious Diseases, Heidelberg University Hospital, Im Neuenheimer Feld 324, 69120, Heidelberg, Germany. E-mail address: Claudia.denkinger@uni-heidelberg.de (C.M. Denkinger).

Joint first authors.
} the CC BY-NC-ND license (http://creativecommons.org/licenses/by-nc-nd/4.0/). 


\section{Materials and methods}

We performed a large-scale, pragmatic implementation study of Ag-RDTs in the context of a universal screening programme at one of Germany's largest tertiary care hospitals (Heidelberg University Hospital, Germany) serving over 100000 inpatients and 1.3 million outpatients per year [11]. The study was conducted between 20 September 2020 and 7 March 2021 during Germany's second wave of COVID-19. Over the duration of the study, SARS-CoV-2 incidence in the region served by the hospital ranged from 11.6 cases per 100000 inhabitants in the last 7 days (23 September) to 254.2 (22 December) [12]. Patients without SARS-CoV-2-associated symptoms presenting for elective procedures, including outpatient treatment requiring close contact or longer presence (e.g., dialysis), were screened with an Ag-RDT. Additionally, depending on setting and local SARS-CoV-2 infection dynamics, other external personnel (e.g., craftspeople, visitors, translators) were similarly screened. The test used was the STANDARD Q COVID-19 Ag Test (SD Biosensor, Inc. Gyeonggi-do, Korea), a WHO recommended and independently validated instrument-free lateral flow assay for SARS-CoV-2 detection [13-15] performed at point of care using the nasopharyngeal swabs provided in the kit. Prior to screening initiation, nursing staff were trained to perform the test and had to pass a competency test implemented by members of the hospital hygiene department. In patients with a positive Ag-RDT, an additional nasopharyngeal swab was collected for confirmatory SARS-CoV-2 PCR using one of the following commercially available PCRs: the SARS CoV-2 assay from TibMolbiol (Berlin, Germany), the Allplex SARS-CoV-2 Assay from Seegene (Seoul, South Korea) or the Abbott (Illinois, USA) RealTime 2019-nCoV assay. Ag-RDT results were confirmed with PCR in selected departments (e.g., haematology) prior to ward admission, prior to planned procedures associated with high levels of aerosol production, when a patient developed SARS-CoV-2 associated symptoms, or for contact screening when a case was detected on hospital wards. To analyse diagnostic yield of Ag-RDTs and false positives, we systematically extracted results of the Ag-RDTs performed from the hospital internal laboratory system, as well as results of PCR tests (with CT values) that were done within 5 days after Ag-RDT screening. Positive predictive value (PPV), sensitivity, and 95\% confidence intervals were computed using the confirmatory PCR result as the reference standard.
Because tests performed in the same individual are expected to be correlated, sensitivity and predictive values were calculated on individual patient rather than test level. Analysis was conducted using R v4.0.3 (The R Foundation). The ethics review board at Heidelberg University approved this study (S-811/2020).

\section{Results}

Between 20 September 2020 and 7 March 2021, 49542 Ag-RDTs were performed in 27421 asymptomatic individuals. Of the total 27421 patients tested, 19712 (72\%) were only tested once. Ag-RDTs were positive in 222 individuals and $49320 \mathrm{Ag}$-RDTs were negative in 27199 individuals. Out of the 222 individuals with positive AgRDTs, 196 (88.3\%) were also tested using PCR in-house. The PPV for the Ag-RDTs was 170/196 (86.7\%, 95\% CI 81.2-91.1\%). Among patients with a positive confirmatory PCR performed within 5 days of a positive Ag-RDT, the median CT value was 19 (interquartile range, IQR 15-24, Fig. 1). Additionally, 7165 PCRs were negative within 5 days of a negative RDT.

Of 27421 patients with a negative Ag-RDT, 94 had a positive PCR in the 5 days following the Ag-RDT. The median number of days between the tests was 1 (IQR $0-1$ ). Based on these false-negative cases identified via PCR, the overall sensitivity of the Ag-RDT can be estimated to be $170 / 264$ (64.4\%, 95\% CI 58.3-70.2). The median CT value of patients who were missed using Ag-RDTs was 33 (IQR 29-35). In total, only $12 / 94$ (12.8\%) Ag-RDT false-negative patients had a PCR with a CT value $<25$, and $10 / 12$ were identified on the same or following day.

\section{Discussion}

This pragmatic implementation study of a universal screening programme of asymptomatic patients at a tertiary care hospital where quick turnaround and detection of cases with high risk of secondary transmission were essential showed the benefit of AgRDTs identifying SARS-CoV-2-infected persons who would have otherwise entered a high-risk environment leading to potential secondary transmission. The sensitivity estimated in this study is higher than that observed in other studies of asymptomatic infections [7]. Although the data on accuracy in asymptomatic patients is limited, we acknowledge that the sensitivity estimate is
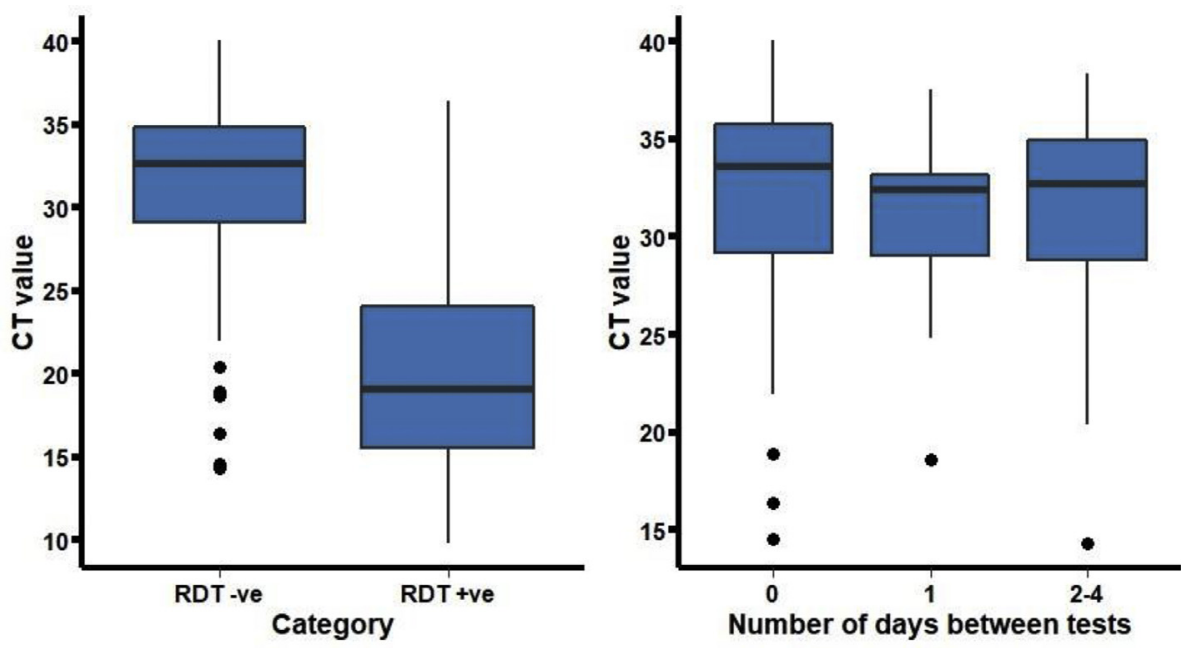

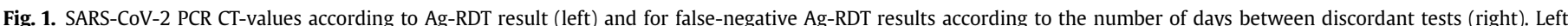

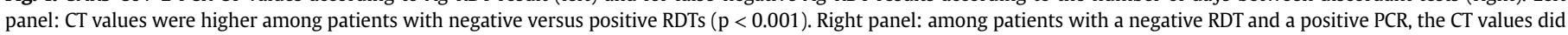
not differ between the different groups ( $p=0.339$ ). 
likely an overestimate (i.e., at most $64.4 \%$ ), as not all patients with asymptomatic infections were PCR tested in this pragmatic study. However, as viral load kinetics have been confirmed to be largely similar in asymptomatic and symptomatic patients, judging from performance in symptomatic patients, one would expect to capture most infections in the first week of illness in asymptomatic persons when viral load is high and thus prevent most secondary transmissions $[7,16,17]$. This is supported by the few new cases observed in the hospital during the study period. The imperfect sensitivity however highlights the need for the continuation of other protective measures. Furthermore, authorities have recommended against clinical extrapolation of infectiousness based exclusively on CT [18]. Missed cases with CT $<25$ on PCR testing were identified within 24 hours and are likely attributable to a negative Ag-RDT in the early phase of disease when the viral load is increasing rapidly [19]. Our study also showed a very high PPV of the Ag-RDT, thus confirming the high reliability of a positive result from an Ag-RDT shown in accuracy studies [7].

To the best of our knowledge, this is the first large-scale implementation study of a universal screening programme of asymptomatic individuals to analyse the diagnostic yield of SARSCoV-2 Ag-RDTs. The central limitation of this study, inherent to a pragmatic implementation study, is the limited confirmatory PCR testing for negative Ag-RDTs, and potential selection bias associated with confirmatory testing being only performed in high-risk or subsequently symptomatic patients. Additionally, the delay in confirmatory PCR-based testing merits caution when interpreting the sensitivity and specificity estimates.

In conclusion, this study provides evidence that an Ag-RDT can be a suitable test for large-scale universal screening in a hospital setting and adds the important component of a public health test to our diagnostic armamentarium. Even as vaccination rates continue to increase, given the emergence of novel virus variants, resulting immune escape despite vaccination, and the high risk associated with undetected infections in clinical settings, we expect universal screening to remain necessary as the pandemic progresses.

\section{Transparency declaration}

Mr Wachinger, Dr Olaru, Mrs Horner, Dr Schnitzler and Dr Heeg have nothing to disclose; Dr Denkinger reports grants from Ministry of Science, Research and the Arts, State of Baden-Wuerttemberg, Germany, during the conduct of the study. The authors did not receive any industry support for conducting this study. Within the previous 3 years, C.M.D. has received support from SAP SE, and P.S. has received support from Janssen-Cilag, MSD Sharp \& Dohme and RAM Group DE GmbH for work not related to this study. J.W., I.D.O., S.H. and K.H. have nothing to disclose. This study was funded in part by the Ministry of Science, Research and the Arts, State of BadenWuerttemberg, Germany, as well as hospital-internal funds.

\section{Author contributions}

J.W., K.H. and C.M.D. conceptualized the study. I.D.O. analysed the data, supported by J.W., S.H., P.S. and C.M.D. J.W., I.D.O. and
C.M.D. drafted the manuscript. All co-authors critically revised and approved the final version of the research note.

\section{References}

[1] Moghadas SM, Fitzpatrick MC, Sah P, Pandey A, Shoukat A, Singer BH, et al. The implications of silent transmission for the control of COVID-19 outbreaks. Proc Natl Acad Sci USA 2020;117:17513-5.

[2] World Health Organization. SARS-CoV-2 antigen-detecting rapid diagnostic tests: an implementation guide. 2020 (cited 9 July 2021). Available from: https://www.who.int/publications/i/item/9789240017740.

[3] Smith DRM, Duval A, Pouwels KB, Guillemot D, Fernandes J, Huynh B-T, et al. Optimizing COVID-19 surveillance in long-term care facilities: a modelling study. BMC Med 2020;18:386.

[4] Peeling RW, Olliaro P. Rolling out COVID-19 antigen rapid diagnostic tests: the time is now. Lancet Infect Dis 2021;21:1052-3.

[5] Johnson-León M, Caplan AL, Kenny L, Buchan I, Fesi L, Olhava P, et al. Executive summary: it's wrong not to test: the case for universal, frequent rapid COVID19 testing. E Clin Med 2021;33:100759.

[6] Dinnes J, Deeks JJ, Berhane S, Taylor M, Adriano A, Davenport C, et al. Rapid, point-of-care antigen and molecular-based tests for diagnosis of SARS-CoV-2 infection. Cochrane Database Syst Rev 2021:3:CD013705.

[7] Brümmer LE, Katzenschlager S, Gaeddert M, Erdmann C, Schmitz S, Bota M, et al. The accuracy of novel antigen rapid diagnostics for SARS-CoV-2: a living systematic review and meta-analysis. medRxiv 2021:2021.02 26.21252546. Available from: https://www.medrxiv.org/content/10.1101/2021.02.26. 21252546v1.full.pdf.

[8] Boehme C, Hannay E, Sampath R. SARS-CoV-2 testing for public health use: core principles and considerations for defined use settings. Lancet Glob Health 2021;9:e247-9.

[9] Larremore DB, Wilder B, Lester E, Shehata S, Burke JM, Hay JA, et al. Test sensitivity is secondary to frequency and turnaround time for COVID-19 screening. Sci Adv 2021;7:eabd5393.

[10] Peeling RW, Olliaro PL, Boeras DI, Fongwen N. Scaling up COVID-19 rapid antigen tests: promises and challenges. Lancet Infect Dis 2021. https://doi.org/10.1016/S1473-3099(21)00048-7.

[11] Heidelberg University Hospital. Annual report 2019. 2020 (cited 9 July 2021). Available from: https://report.ukhd-mfhd.de/2019/.

[12] Landratsamt Rhein-Neckar-Kreis. Fallzahlen aus dem Rhein-Neckar-Kreis und dem Stadtgebiet Heidelberg. 2021 (cited 5 July 2021). Available from: https://www.rhein-neckar-kreis.de/start/landratsamt| coronavirus+fallzahlen.html.

[13] Berger A, Nsoga MTN, Perez-Rodriguez FJ, Aad YA, Sattonnet-Roche P, GayetAgeron A, et al. Diagnostic accuracy of two commercial SARS-CoV-2 antigendetecting rapid tests at the point of care in community-based testing centers. PLoS ONE 2021:16:e0248921.

[14] Krüger LJ, Gaeddert M, Köppel L, Brümmer LE, Gottschalk C, Miranda IB, et al. Evaluation of the accuracy, ease of use and limit of detection of novel, rapid, antigen-detecting point-of-care diagnostics for SARS-CoV-2. medRxiv. 2020. 2020.10.01.20203836. Available from: https://www.medrxiv.org/content/10. 1101/2020.10.01.20203836v1.full.pdf.

[15] World Health Organization. WHO emergency use assessment coronavirus disease (COVID-19) IVDs: public report. 2020 (cited 8 July 2021). Available from: https://www.who.int/diagnostics_laboratory/eual/201019_final_pqpr_ eul_0563_117_00_standard_q_covid19_ag_test.pdf?ua=1.

[16] Lee S, Kim T, Lee E, Lee C, Kim H, Rhee H, et al. Clinical course and molecular viral shedding among asymptomatic and symptomatic patients with SARSCoV-2 infection in a community treatment center in the Republic of Korea. JAMA Intern Med 2020;180:1447-52.

[17] Jones TC, Biele G, Mühlemann B, Veith T, Schneider J, Beheim-Schwarzbach J, et al. Estimating infectiousness throughout SARS-CoV-2 infection course. Science 2021;373:eabi5273.

[18] Centers for Disease Control and Prevention. Clinical questions about COVID19: questions and answers. 2021 (cited 5 July 2021). Available from: https://www.cdc.gov/coronavirus/2019-ncov/hcp/faq.html\#Testing,-Isolation,-and-Quarantine-for-Persons-Who-Have-Recovered-from-PreviousSARS-CoV-2-Infection.

[19] Kissler SM, Fauver JR, Mack C, Olesen SW, Tai C, Shiue KY, et al. Viral dynamics of acute SARS-CoV-2 infection and applications to diagnostic and public health strategies. PLOS Biol 2021;19:e3001333. 